\title{
SESELIL. (UMBELLIFERAE) TÜRLERİNİN KİMYASAL BİLEŞİMİ VE BİYOLOJİK AKTİVITELERİ
}

\section{CHEMICAL CONSTITUENTS AND BIOLOGICAL ACTIVITIES OF SESELIL.} (UMBELLIFERAE) SPECIES

\author{
Alev TOSUN* Nazire ÖZKAL \\ Ankara University, Faculty of Pharmacy, Department of Pharmacognosy, \\ 06100 Tandoğan, Ankara, TURKEY
}

\section{ÖZET}

Bu derlemede Seseli L. türlerinin taşıdı̆̆ı, kumarinler başta olmak üzere, terpenik yapıda bileşikler, fenilpropanoitler ve diğer bazı bileşikler ile bu türler üzerinde yapılmış bazı aktivite çalışmalarına ve etkilerine değinilmiştir.

Anahtar kelimeler: Umbelliferae, Seseli L., kimyasal bileşim, biyolojik aktivite

\begin{abstract}
in this review, the species of Seseli L. have been evaluated regarding to the content of coumarins as well as the terpenoid compounds, phenylpropanoids and other different compounds. The biological activities ofthese species and some compounds, also their usage was discussed.
\end{abstract}

Key words: Umbelliferae, Seseli L., chemical constituent, biological activity

\section{GİRİŞ}

Genellikle 1lıman bölgelerde yayılış gösteren yaklaşık 455 cins ve 3600-3751 kadar türe sahip olan Umbelliferae familyası, Türkiye'de 97 cins ve 430 civarında tür ile temsil edilmektedir (1-6).

Umbelliferae familyasmdaki Seseli L., Avrupa ve Asya'da 80 civarında tür ile temsil edilen, oldukça yükseklerde, genellikle kayalarda yetişen çoğunlukla otsu bitkilerdir. Türkiye'de ise tür ve tür altı seviyesinde 12 takson ile temsil edilmektedir (6-10).

Seseli L., Hippocrates tarafından Umbelliferae familyasının oldukça çekici görünüşleri olan bazı üyeleri için kullanılmış eski bir Yunan ismidir (11).

Literatür incelendiğinde, Seseli L. türlerine ait kimyasal çalışmaların daha çok kumarinler üzerinde yoğunlaştığı (Tablo 1-5), bunun yanında farklı etken madde grupları üzerinde yapılmış bazı çalışmaların da bulunduğu (Tablo 6-8) görülmektedir. 
Seseli L. türlerinde varlıkları saptanmış ve izole edilmiş kumarin türevi bileşikler yapısal farklılıklarına göre 6 grup halinde toplanarak aşă̆ıdaki tablolarda verilmiştir (Tablo 1-5).

Tablo 1. Seseli L. türlerinden izole edilen basit kumarin yapısında bazı bileşikler

Basit Kumarinler

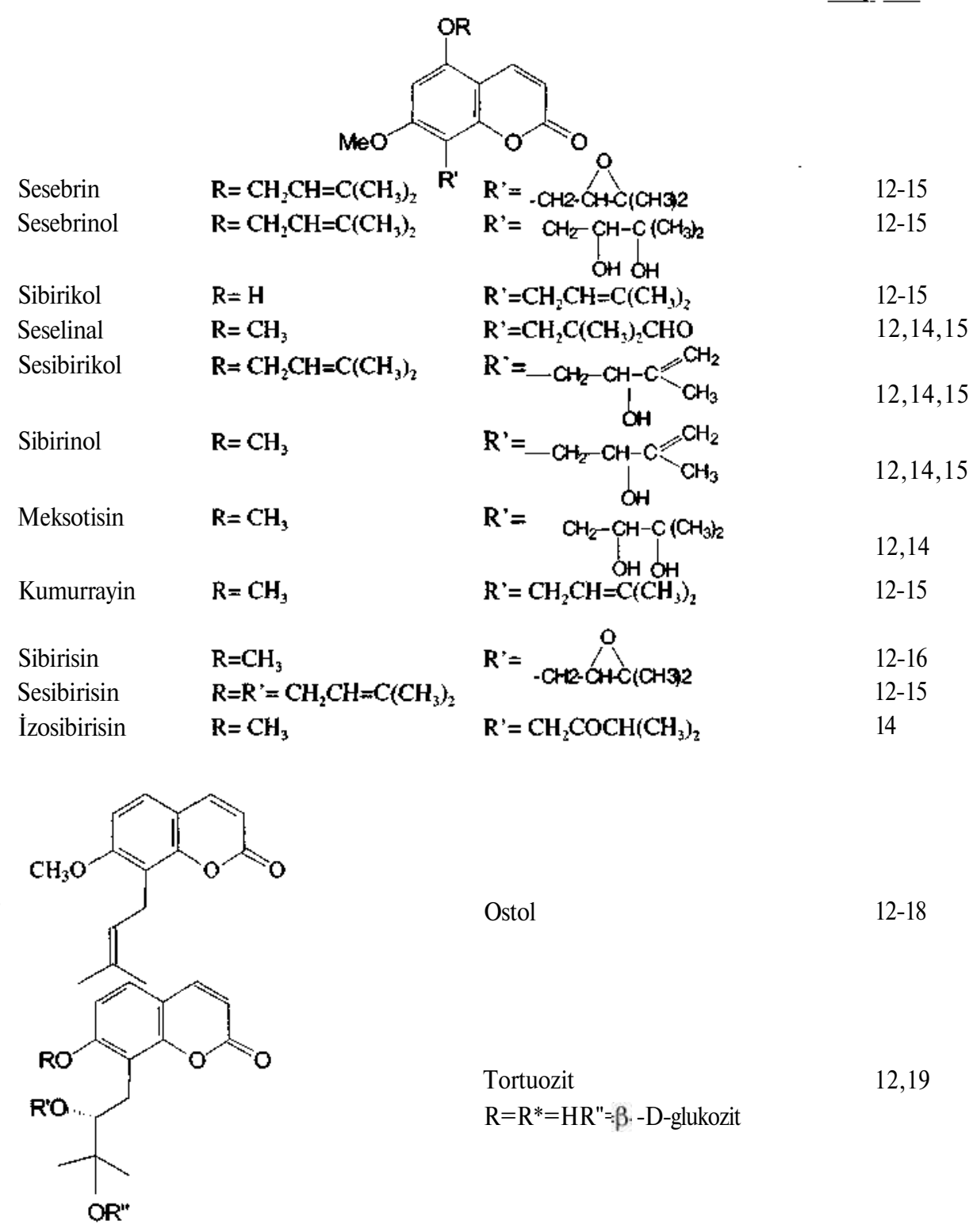

\section{$\underline{\underline{\text { Kaynak }}}$}

$12-15$

(1)

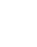


Ankara Ecz. Fak. Derg., 32 (4) 269-284,2003

Tablo 2. Seseli L. türlerinden izole edilen angular furokumarin yapısında bazı bileşikler

Angular Furokumarinler

$\underline{\underline{\text { Kavnak }}}$<smiles>[R20]C1c2c(ccc3ccc(=O)oc23)O[C@@H]1C(C)(C)OC(C)=O</smiles>

$\begin{array}{ll}\text { İzopösenidin } & \mathrm{R}=\mathrm{COCH}=\mathrm{C}\left(\mathrm{CH}_{3}\right)_{2} \\ \text { Libanotin } & \mathrm{R}=\mathrm{COC}\left(\mathrm{CH}_{3}\right)=\mathrm{CHCH}_{3}\end{array}$<smiles>COc1cc2ccc(=O)oc2c2cc([SeH])oc12</smiles><smiles>COc1cc2occc2c2oc(=O)ccc12</smiles> 
Tablo 3. Seseli L. türlerinden izole edilen linear furokumarin yapısında bazı bileşikler

Linear Furokumarinler

(2’S)-Rutaretin-1'"-sülfat

Deltoin

Kampesenin (Rutarin)

Psoralen

İmperatorin

İzoimperatorin

Alloizoimperatorin

Bergapten

Ksantotoksin

Ksantotoksol

İzopimpinellin

Fellopterin

Oksipösedanin hidrat<smiles>[R]C(C)(C)[C@H]1Cc2cc3ccc(=O)oc3cc2O1</smiles>

$\mathrm{R}=\mathrm{SO}_{3} \mathrm{~K}$<smiles>[R]C(C)(C)[C@H]1Cc2cc3ccc(=O)oc3cc2O1</smiles>

$\mathrm{R}=\mathrm{COCCH}_{2}=\mathrm{CHCH}_{3}$<smiles>[R]c1c2ccc(=O)oc2c([R])c2ccoc12</smiles>

$$
\begin{array}{ll}
\mathrm{R}=\mathrm{H} & \mathrm{R}^{\prime}=\mathrm{H} \\
\mathrm{R}=\mathrm{OCH}_{2} \mathrm{CH}=\mathrm{C}\left(\mathrm{CH}_{3}\right)_{2} & \mathrm{R}^{\prime}=\mathrm{H} \\
\mathrm{R}=\mathrm{H} & \mathrm{R}^{\prime}=\mathrm{OCH}_{2} \mathrm{CH}=\mathrm{C}\left(\mathrm{CH}_{3}\right)_{2} \\
\mathrm{R}=\mathrm{CH}_{2} \mathrm{CH}=\mathrm{C}(\mathrm{CH},)_{2} & \mathrm{R}^{\prime}=\mathrm{OH} \\
\mathrm{R}=\mathrm{H} & \mathrm{R}^{\prime}=\mathrm{OCH}_{3} \\
\mathrm{R}=\mathrm{OCH}_{3} & \mathrm{R}^{\prime}=\mathrm{H} \\
\mathrm{R}=\mathrm{OH} & \mathrm{R}^{\prime}=\mathrm{H} \\
\mathrm{R}=\mathrm{OCH}, & \mathrm{R}^{\prime}=\mathrm{OCH}, \\
\mathrm{R}=\mathrm{OCH}_{2} \mathrm{CH}=\mathrm{C}\left(\mathrm{CH}_{3}\right)_{2} & \mathrm{R}^{\prime}=\mathrm{OCH}, \\
\mathrm{R}=\mathrm{H} & \mathrm{R}^{\prime}=\mathrm{CH}_{2}-\mathrm{CH}-\mathrm{C}\left(\mathrm{CH}_{3}\right)_{2} \\
&
\end{array}
$$

$\underline{\underline{\text { Kaynak }}}$
12,22

12,23

24

\section{3}

25

12-16,18

$12,21,24$

$12,13,21$

$12-16,18,25$

$12,14,15,21,25$

12,21

$12.14,15,21$

$12,13,21$

12,21 
Tablo 4. Seseli L. türlerinden izole edilen angular piranokumarin yapısındaki bazı bileşikler<smiles>CC1(C)C=Cc2c(ccc3ccc(=O)oc23)O1</smiles>

\section{Seselin}<smiles>[R10]OC1c2c(ccc3ccc(=O)oc23)OC1(C)C</smiles>

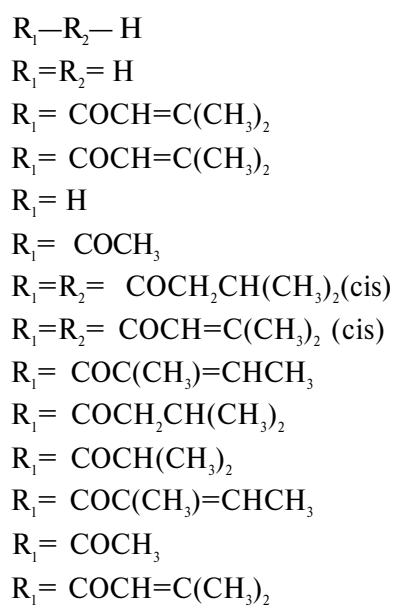

$\mathrm{R}_{1}=\mathrm{COCH}_{2} \mathrm{CH}\left(\mathrm{CH}_{3}\right)_{2}$

$\mathrm{R}_{1}=\mathrm{COCH}\left(\mathrm{CH}_{3}\right)_{2}$

$\mathrm{R}_{1}=\mathrm{COC}\left(\mathrm{CH}_{3}\right)=\mathrm{CHCH}_{3}$

$\mathrm{R}_{1}=\mathrm{COCH}_{3}$

$\mathrm{R}_{1}=\mathrm{COCH}=\mathrm{C}\left(\mathrm{CH}_{3}\right)_{2}$

12,26

12,26

$\mathrm{R}_{2}=\mathrm{COC}\left(\mathrm{CH}_{3}\right)=\mathrm{CHCH}_{3}$ (cis)

$12,26,27$

$\mathrm{R}_{2}=\mathrm{COCH}_{3}$ (Bokkonin)

12,18

$\mathrm{R}_{2}=\mathrm{H}$

12,18

$\mathrm{R}_{2}=\mathrm{COC}\left(\mathrm{CH}_{3}\right)=\mathrm{CHCH}_{3}$ (Pteriksin)

12,25

$\mathrm{R}_{2}=\mathrm{COC}\left(\mathrm{CH}_{3}\right)=\mathrm{CHCH}_{3}$<smiles>[R10]O[C@@H]1[C@@H](OCC)c2c(ccc3ccc(=O)oc23)OC1(C)C</smiles>

$\mathrm{R}_{1}=\mathrm{R}_{2}=\mathrm{COCH}=\mathrm{C}\left(\mathrm{CH}_{3}\right)$,

$\mathrm{R}_{\mathrm{l}}=\mathrm{COCH} 3$ 
Tablo 4. Seseli L. türlerinden izole edilen angular piranokumarin yapısındaki bazı bileşikler (Devamı)

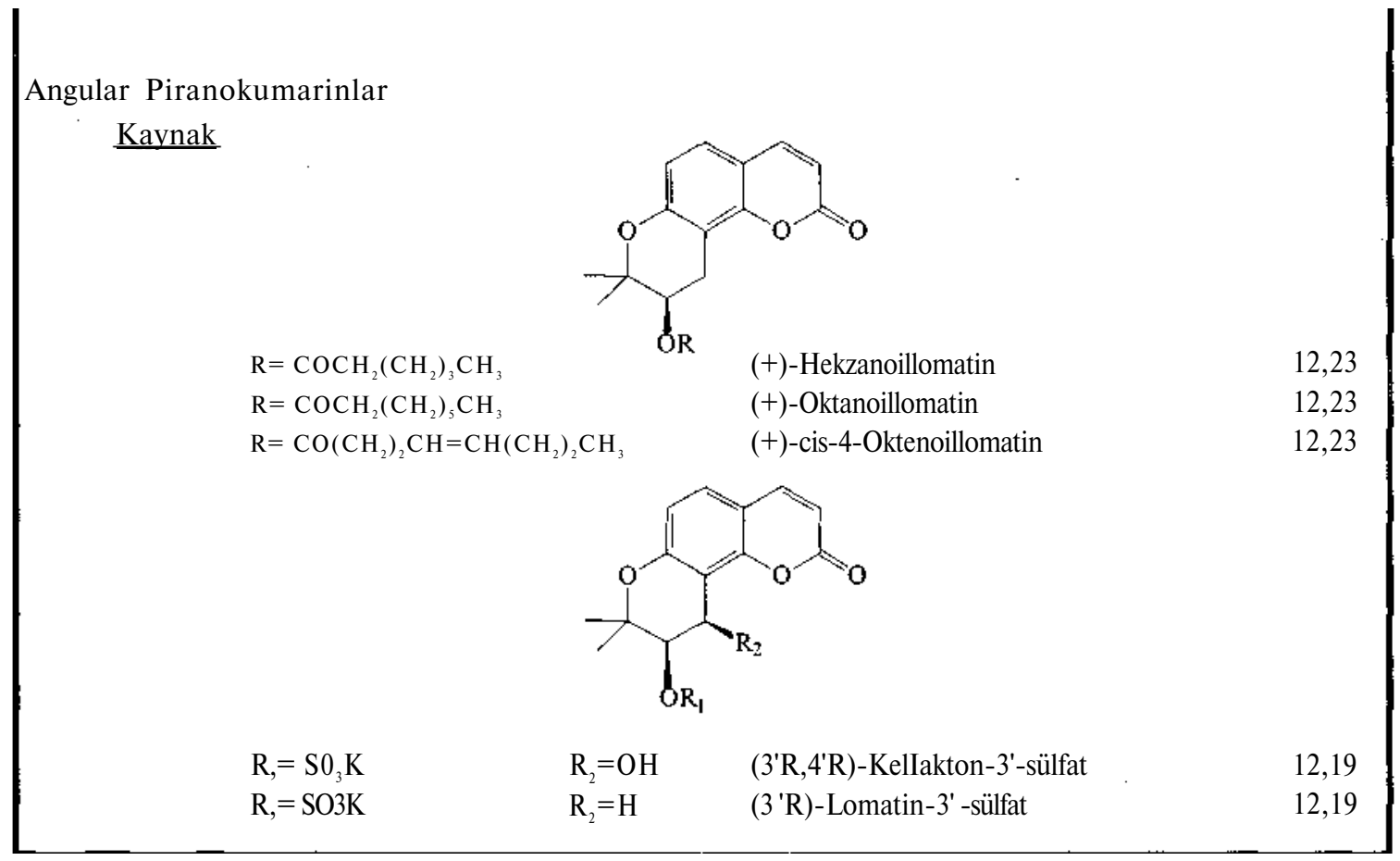

Tablo 5. Seseli L. türlerinden izole edilen linear piranokumarin yapısında bazı bileşikler

Linear Piranokumarinler

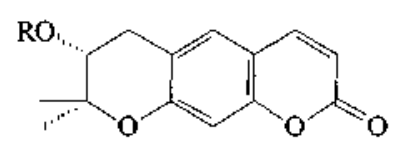

$\mathrm{R}=\mathrm{COCH}=\mathrm{CH}\left(\mathrm{CH}_{3}\right)_{2} \quad(-)-2^{\prime}$-senesioiloksi-l', 2'-dihidroksantiletin

12,19

$\mathrm{R}=\mathrm{COCH}_{2} \mathrm{CH}\left(\mathrm{CH}_{3}\right)_{2}$

(-)-2'-izovaleriloksi-I $\backslash 2$ '-dihidroksantiletin

Farklı türlerin çeşitli kısımları üzerinde yapılan çalışmalarda diğer bazı etken madde gruplarına ait bileşiklerin varlığına da rastlanmıştır.

Literatürde Seseli türlerinin uçucu yağı ve terpenik bileşikleri ile yapılmış çok az sayıda çalışma bulunmaktadır. Güneydoğu İspanya'nın denize yakın kayalıklarından toplanan ve endemik bir tür olan Seseli vayredanum L. \{Athamantha vayredana) üzerinde aynı araştırmacılar tarafından yayınlamış iki farklı çalışmaya rastlanmıştır. Bu çalışmalara göre bu türün topraküstü kısmının Soxhlet ekstraksiyonu ile hazırlanan hekzanlı ekstresinden seskiterpen yapısında pek çok bileşik (Tablo 6) izole edilmiştir $(28,29)$. 
Tablo 6. Seseli L. türlerinde bulunan seskiterpen yapısındaki bazı bileşikler $(28,29)$

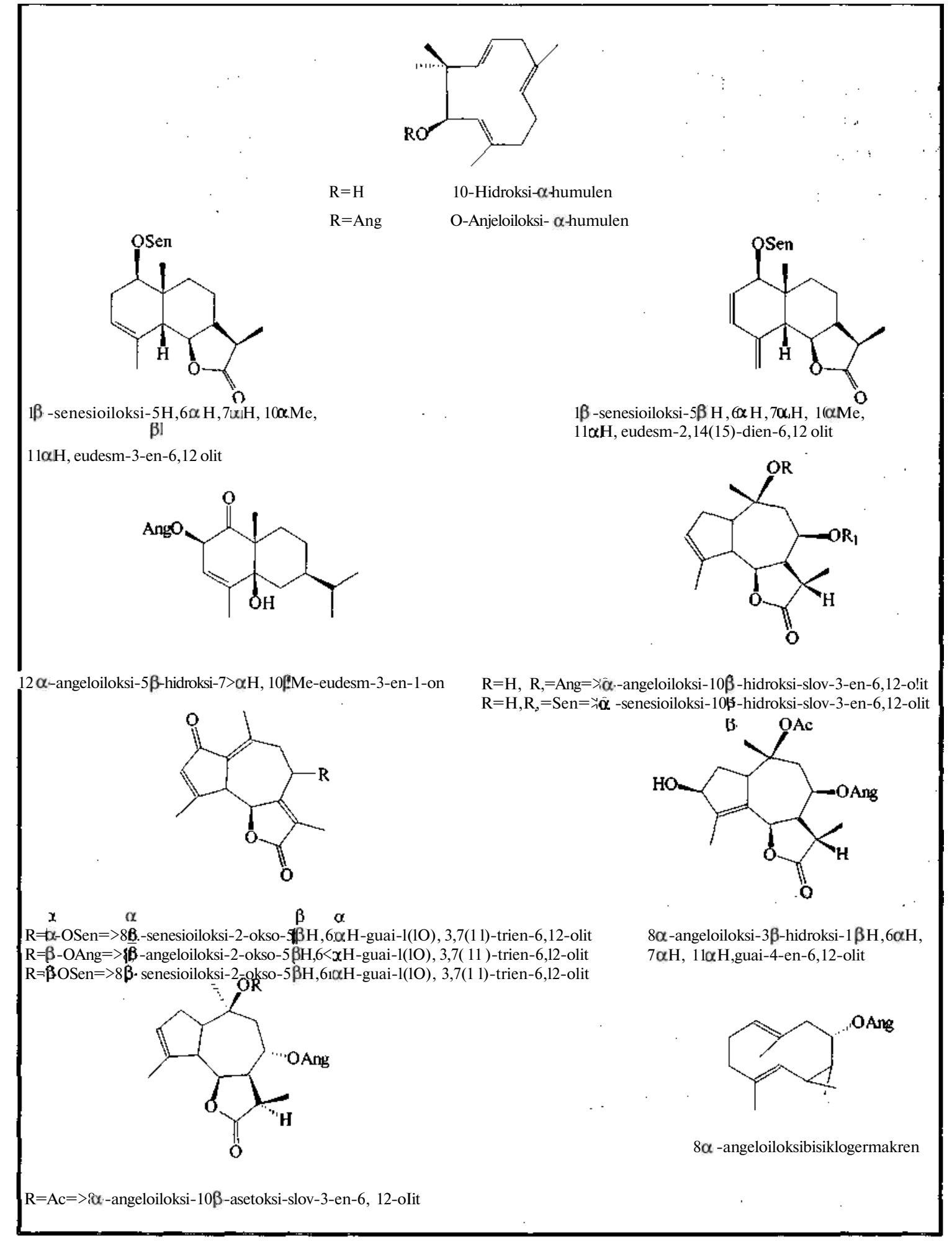


Seseli türlerinin uçucu yağları ile yapılmış birkaç çalışmadan biri de, Seseli elatum L. topraküstü kısmının uçucu yă̆ı ile olan çalışmadır. Seseli elatum subsp. gouanii (Koch) P.W.Ball. $\left(\mathrm{Se}_{\mathrm{G}}\right)$, petrol eteri kullanılarak perkolasyonla ekstre edilmiş, solvan uzaklaştırıldıktan sonra, artık buhar distilasyonuna tabi tutulmuş ve susuz $\mathrm{Na}_{2} \mathrm{~S}_{4}$ ile kurutulduktan sonra uçucu yağ miktarı \% 0.8 olarak bulunmuştur. Bu uçucu yağın, analiz neticesinde seskiterpen hidrokarbonlarca çok zengin olduğu görülmüştür. Bu seskiterpen hidrokarbonlar arasında Pkaryofillen, majör bileşik olarak bulunurken bunu izleyen 8-kadinen ve P-bisabolen de önemli miktarlarda gözlenmiştir. Monoterpen bileşimini ise, a-pinen, P-mirsen oluşturmuştur. $\mathrm{Se}_{\mathrm{G}}{ }^{\prime}$ nin yapraklarından elde edilen uçucu yağın, P-mirsen, P-karyofillen, a-pinen ve p-simen gibi bileşikleri yüksek oranda taşıdı̆̆ı; dallarından elde edilen uçucu yağın monoterpen hidrokarbonlarca zengin olduğu; çiçeklerinin uçucu yağında ise başlıca seskiterpenlerin bulunduğu belirtilirken, meyvalardan elde edilen uçucu yağın hemen hemen tamamının seskiterpen hidrokarbonlardan oluştuğu, P-karyofillen ve kalakoren tipi seskiterpen hidrokarbonların da önemli miktarda bulunduğu ifade edilmiştir (30).

Özbekistan'da yetişen bir tür olan Seseli macrophyllum Regel \& Schmalh [Athamantha macrophylla Korov., Medisia macrophylla (Regel \& Schmalh) Pimen.]'un topraküstü kısmından hidrodistilasyonla \% 0.07 oranında uçucu yağ elde edilmiş, bu uçucu yağın, GC-MS analizi sonunda, başlıca \% 27.23 p-simen, \% 15.13 timol, \% 12.52 karvakrol, \% 8.17 pulegon, \% 7.10 mirsen ve oc-fellandren'den oluştuğu anlaşılmıştır (31).

Diğer bir çalışmada Seseli campestre Besser'in, meyva ve herbası üzerinde gerçekleştirilmiştir. Clevenger apareyi kullanılarak yapılan distilasyonda uçucu yağ miktarı meyvalarında \% 1.5, toprak üstü kısmında ise \% 1.0 olarak tespit edilmiştir. Uçucu yağların GC-MS analizleri sonucu başlıca bileşiklerinin a-pinen ve daha önce Peucedanum palustre (L.) Moench'de de varlığı bilinen (E)-seskilavandulol olduğu saptanmıştır. Toprak üstü kısımlarında ve meyvalarda a-pinenin sirasiyla $\% 35.8$ ve \% 26.2; (E)-seskilavandulolün de sirasiyla \% 3.2 ve \% 11.8 oranlarında bulunduğu belirtilmiştir. S. tortuosum'un uçucu yağında ise \% 35.9 apinen, \% 8.8 sabinen, \% 8.4 (E)- seskilavandulol ve \% 7.0 P-pinen bulunduğu görülmüştür (32, 33).

Bir diğer çalışmada İspanya'da endemik bir tür olan Seselifarreynii Molero \& Pujadas'ın taze yapraklarının dietileterli ekstresinin, GC-MS analizi sonucunda seskiterpenik bileşiklerin yanında monoterpenler, tetradesilasetat, tetrakozanol, hekzakozanol, oktakozanol gibi bileşiklere de rastlanmıştır (34).

Fenilpropanoit yapısında bileşiklerin varlığı da yine Seseli vayredanum L.'un topraküstü kısmının hekzanlı ekstresinde miristisin, öjenolmetileter, elemisin, latifolon, eritro-1-hidroksi-2anjeloiloksi-3' ,4'-metilendioksi-5'-metoksi-1 -fenilpropan, treo-1 -hidroksi-2-anjeloiloksi-3' ,4'metilendioksi-5'-metoksi-1-fenilpropan, laserin ve 2-epilaserin gibi bileşiklerin izole edilip saptandığı belirtilmektedir $(28,29)$. Bu grup bileşiklere ait bazı örnekler Tablo 7'de verilmiştir.

Kinonik bileşiklerin izolasyonuna ait sadece bir çalışmaya rastlanmış olup, bu da İspanya'da endemik bir tür olan Seseli farreynii Molero \& Pujadas üzerinde yapılmış ilk kimyasal araştırmadır. $\mathrm{Bu}$ araştırma sonunda, bitkinin taze yapraklarının dietileterli ekstresinin silikajel 
üzerinde yapılmış kolon kromatografisi ve petroleterketilasetat elüsyonu ile 7demetilplastokromenol-2, seskiterpen kinon (Ligusticum chuangxiong'dan izole edilip 2farnesil-6-metil-benzokinon olarak isimlendirilmiş bileşik) ve bir hidrokinon (Tablo 8) izole edilerek yapıları aydınlatılmıştır (34).

Tüm bu bileşiklerin dışında, Seseli sibiricum Benth'un topraküstü kısmının, hekzan ile ekstraksiyonu sonucu elde edilen yağlı artığın, nötral $\mathrm{A}_{2} \mathrm{O}_{3}$ ile kromatografiye edilmesi sonucu, n-hekzan, n-hekzan:benzen, benzen: kloroform ve kloroform ile devam eden elüsyon sisteminde, kumarin bileşikleri yanında sinnamik asit türevi olan sesebrinik asitin (Tablo 8) izole edildiği belirtilmiştir (15).

Tablo 7. Seseli L. türlerinde bulunan fenilpropanoit yapısındaki bazı bileşikler $(28,29)$<smiles>COc1cc(C(OC(C)=O)C(C)OC)cc2c1OCO2</smiles>

Eritro-1-asetoksi-2-anjeIoiloksi-3', 4'metilendioksi-5' -metoksi-1 -fenilpropan

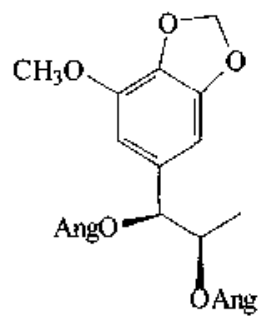

2-Epilaserin<smiles>COc1cc(C(OC(C)=O)C(C)O[Na])cc2c1OCO2</smiles>

Treo-1-asetoksi-2-anjeloiloksi-3',4'metilendioksi-5'-metoksi-1 -fenilpropan

1-Hidroksi-3',4'-metilendioksi-2',5'dimetoksi-1 -fenilpropan

$\mathrm{HO}$<smiles>CCC(O)c1cc(OC)c2c(c1OC)OCO2</smiles>

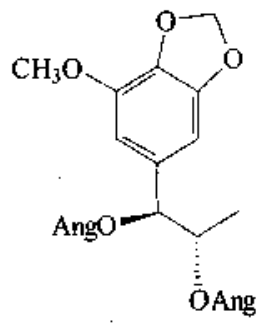

Laserin 
Tablo 8. Seseli L. türlerinde rastlanan diğer bazı bileşiklere örnekler $(15,34)$

HO<smiles>CC(C)=CCC/C(C)=C/CCC1(C)C=Cc2cccc(C)c2O1</smiles>

7-Demetilplastokromenol-2 (Kromenol)

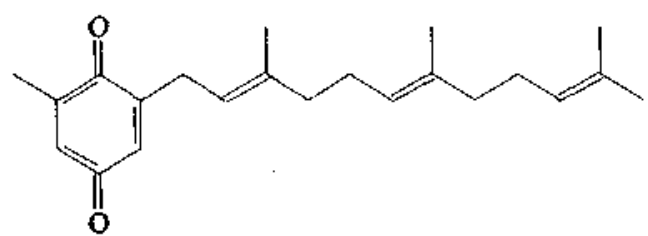

Seskiterpen kinon (2-Famesil-6-metil-benzokinon)<smiles>COc1cc(OCC(C)C)c2c(c1/C=C\C(=O)O)OC(C(C)(C)CO)C2</smiles>

Sesebrinik asit

\section{Seseli L.Türlerine Ait Biyoaktivite Çalışmaları}

\section{Antimikrobiyal Etki}

Seseli libanotis, Ligusticum stewartii, Pycnocycla aucheriana'dan elde edilen uçucu yağların Staphylococcus aureus, Escherichia coli, Shigella dysanteriae, Vibrio cholerae ve Salmonella typi gibi patojen bakterilere kaşı etkileri incelenmiştir. Seseli libanotis'in uçucu yă̆ının, özellikle Staphylococcus aureus'a karşı çok etkili olduğu bulunmuştur (9).

Pek çok uçucu yağın fungitoksik etkiye sahip olduğu da bilinmektedir. Seseli indicum W. \& A.'un uçucu yağının da diğer bazı uçucu yağlarla karşılaştırıldı̆̆ında daha yüksek bir fungitoksik aktiviteye sahip olduğu gözlenmiştir. Yiyecek olarak kullanılan bazı tohumların (Cicer arietinum L., Cajanus cajan (L.) Mili.'in tohumları gibi) depolanması sonucu meydana gelen mantarlar ile protein, aminoasit, şeker ve nişasta içeriklerinin bozulduğu gözlenmiştir. Bu mantarlara karşı uçucu yağların, bu arada $S$. indicum uçucu yağının da doğal kaynaklı bir koruyucu olabileceği ileri sürülmektedir (35).

Umbelliferae familyasının pek çok türünde bulunmakla birlikte Seseli türlerinde de bulunan angular tip bir piranokumarin türevi olan suksdorfinin anti-HIV etkisi in vitro olarak denenmiş ve etkili olduğu görülmüştür (36). 


\section{Antitümör Etki}

Kumarinlerin antimutajenik ve antikarsinojenik aktiviteleri araştırılmış ve etkili oldukları görülmüştür (37-40). P-388 lemfositiklösemi hücrelerine karşı, 3',4'-dihidroksi-3',4'dihidroseselin türevlerinin sitotoksik etki gösterdikleri bilinmektedir (40). Seseli mairei Wolf.'un köklerinin etanollü ekstresinin fraksiyonlarının sitotoksik etkileri araştırılmış ve KB, P-388, L-1210 tümör hücrelerine karşı sitotoksik etki gösterdiği anlaşılmıştır ( $\left.\mathrm{ED}_{50}<20 \mathrm{ug} / \mathrm{ml}\right)$. Daha sonra etkili fraksiyonun seselidiol içerdiği bulunmuş ve bu poliasetilen türevi bileşik, etkili fraksiyondan izole edilmiştir. $\mathrm{Bu}$ maddenin yapısı çeşitli spektral yöntemlerle aydınlatılmıştır (41).

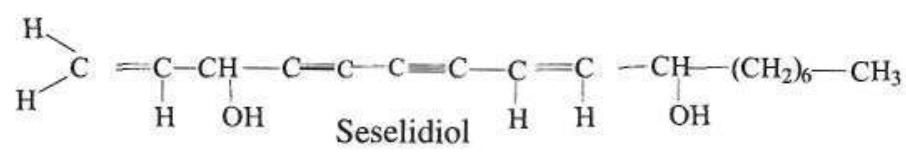

\section{Antienflamatuar Etki}

Çeşitli kumarin türevlerinin antienflamatuar etkisinin olduğu bilinmektedir $(39,40,42,43)$.

Çin'in Yun-Nan ve Si-Chuan bölgelerinde yetişen Seseli mairei Wolf'un kökleri, "Zhu Ye Fang Feng" olarak Çin halkı arasında bilinir. Bu kökler, enflamasyon, şişlik, romatizma, ağrı ve soğuk algınlığına karşı bitkisel şifa olarak kullanılmaktadır (41).

$S$. indicum'un tohumlarından izole edilen seselin'in antienflamatuar etkisinin yanında analjezik, antipiretik ve antikonvülzan etkileri de araştırılmıştır. Bunun sonucunda seselin bileşiğinin doza bağlı olarak gelişen antienflamatuar etkisi, farelerde karrageninle indüklenen akut enflamasyonda gözlenmiştir. Ancak, formaldehitle indüklenen subakut enflamasyonlarda etkili değildir. Bunun yanında, farelerde asetik asit ile indüklenmiş ağrıdan dolayı oluşan kıvranmalarda önemli bir analjezik etkisinin olduğundan da söz edilmektedir $(42,43)$.

\section{Kalsiyum Antagonisti ve Koroner Vazodilatör Etki}

Umbelliferae familyası'nın diğer türlerinde olduğu gibi Seseli türlerinde de kalsiyum antagonisti gibi hareket eden furokumarinler mevcuttur. Böylece kardiyovasküler hastalıkların tedavisinde kullanılmaktadırlar. Ostol ve ksantotoksin ile bir çalışma yapılmış ve ostolün güçlü kalsiyum antagonisti olarak hareket ettiği anlaşılmıştır. Ayrıca (+)-cis-kellakton diesterlerinin de koroner vazodilatatör etkileri oldukça iyi bilinmektedir. S. libanotis'den elde edilen (+)-ciskellakton-sülfatesterleri, dihidropiranokumarinlerin ve dihidrofuranokumarinlerin cAMPfosfodiesterazı inhibe edici özellikleri ile spazmolitik ve koroner vazodilatatör olarak kaydadeğer bir etki gösterdikleri anlaşılmıştır (15, 22,44-46).

Seseli libanotis'in tohumlarının Pakistan'da kan basıncı kontrolü için kullanıldığı da bilinmektedir (9). 


\section{Fotosensibilizan Etki}

Umbelliferae familyasına ait Seseli'de dahil hemen hemen tüm cinslerde furokumarinlere rastlanmıştır. Bu bileşikler fotosensibilizan özelliğe sahiptir. Bu etkilen ile vitiligonun lökodermik lekelerinin tedavisinde ve psöriazis de kullanıldıkları bilinmektedir (21,47-51).

\section{Fototoksik Etki}

Furokumarinler, DNA'nın pirimidin bazına foto bağlanma ile böceklere, protozoalara, mantarlara, bakterilere, virüslere karşı fototoksik özellik gösterirler $(21,51)$.

\section{Böcek Kaçırıcı Etki}

Luvunga scandens, Nordostachys jatamansi ve Seseli indicum'un meyvalarından hidrodistilasyon ile elde edilen uçucu yağların, böcek kaçırıcı etkisi incelenmiştir. Bu uçucu yağlar arasında en yüksek böcek kaçırıcı etki, 5. indicum'un uçucu yağında bulunmuştur (52).

Seseli elatum'a ait alt türlerden elde edilen izopimpinellinin yılanlara karşı öldürücü bir zehir olduğu, ayrıca parazitik solucanlara karşı etkili olup, diüretik bir özelliğe sahip olduğu da literatürde kayıtlıdır (21). Seseli indicum'un tohumlarının da eskiden beri antihelmentik, karminatif, stomaşik ve stimulan özelliklere sahip olduğu yine literatürde kayıtlı olan etkiler arasındadir. (42).

Ayrıca yine halk arasında horozgözü olarak bilinen Seseli tortuosum L.'un meyvalarının emanagog ve karminatif olarak kullanıldığı da literatürde yer almaktadır (53).

\section{SONUÇ}

Çoğu kokulu bitkileri içeren Umbelliferae familyasının bir üyesi olan Seseli L. cinsine ait, 4'ü endemik olmak üzere 12 takson ülkemizde doğal olarak yetişmektedir. Ülkemizde bu cinse ait türlerin incelenmesi ve bilim dünyasına kazandırılması gerektiği yapılan literatür incelemeleri ile anlaşılmıştır.

$\mathrm{Bu}$ türlerle yapılan çalışmaların çoğu kumarinler üzerinde yoğunlaşırken, diğer bir kaç grup bileşik üzerinde de yapılmış çalışmalara rastlanmıştır. Bu bileşikler anlatıldığı üzere terpenik, fenilpropanoit yapıdaki bazı bileşikler ile uçucu yağları kapsamaktadır. Yapılan literatür incelemeleri sonunda Seseli L. türlerinden izole edilen kumarin yapısındaki bileşiklerin çoğunlukla angular tip piranokumarin yapısında olduğu görülmüştür. Kumarin yapısındaki bileşiklerin bilinen bir çok aktivitesi $(21,24,36-38,42,43,45-47,49,51,53-59)$ içinde, angular tip piranokumarinlerin de önemli farmakolojik aktiviteleri ile yer aldıkları yapılan çalışmalarda gösterilmiştir (36, 38,42,43,45, 55, 58-60).

$\mathrm{Bu}$ derleme ile, ülkemizde halk arasında henüz fazla tanınmayan ve yöresel bazı kullanımları $(53,61)$ dışında fazla bir kullanımına da rastlanmayan Seseli L. cinsinin içerdiği bileşikler ve farmakolojik etkileri açısından araştırmaya değer olduğu sonucuna varılmıştır. 


\section{KAYNAKLAR}

1. Crowden, R.K., Harborne, J.B., Heywood, V.H. "Chemosystematics of the Umbelliferae-A General Survey" Phytochemistry, 8, 1963-1984 (1969).

2. Lawrence, G.H.M., Taxonomy of Vascular Plants, The Macmillan Company, New York, p.642-646 (1969).

3. Baytop, A., Farmasötik Botanik Ders Kitabı, Üniv. Yayın No:3637, Fakülte Yayın No:58, İ.Ü. Basımevi ve Film Mekezi, İstanbul, s.220 (1991).

4. Pimenov, M.G., Leonov, M.V., The Genera of Umbelliferae, Whitstable Litho, Whitstable, Kent, Great Britain, (1993).

5. Tanker, N., Koyuncu, M., Coşkun, M., Farmasötik Botanik, A. Ü. Ecz. Fak. Yayınları, Ders Kitapları, No:78, , Ankara Üniv. Basımevi, Ankara, s.313 (1998).

6. Duman, H., "Seseli L." in Flora of Turkey and The East Aegean Islands, Güner, A., Özhatay, N., Ekim, T., Başer, K.H.C. (Eds.) Vol. 11, Edinburgh University Press, Edinburgh,p.141 (2000).

7. Hedge, I.C., Lamond, J.M., "Seseli L." in Flora of Turkey and the East Aegean Islands, Davis, P.H. (Ed.) Vol. 4, Edinburgh University Press, Edinburgh, p.367-372 (1972).

8. Davis, P.H., Mili, R.R., Tan, K., Flora of Turkey and The East Aegean Islands, Vol.10, Edinburgh University Press, Edinburgh, p.150-151 (1988)

9. Syed, M., Chaudhary, F.M., Bhatty, M.K. "Antimicrobial Activity of the Essential Oils of Umbelliferae Family. Part III. Seseli libanotis, Ligusticum stewartii and Pycnocycla aucheriana Oils" Pak. J. Sci. Ind. Res., 32(5), 316-319 (1989).

10. Parolly, G., Nordt, B. "Seseli hartvigii (Apiaceae), a New Name for S. ramosissimum Hartvig \& Strid, with Carpological and Ecological Notes on this Species" Willdonowia, 31,87-93(2001).

11. Hamlyn, P., The Marshall Cavendish, Encyclopedia of Gardening, Vol.19, Garrod and Lofthouse International, London, p.2034 (1969).

12. Barrero, A.F., Herrador, M.M., Arteaga, P. "Cumarinas En Especies Del Genero Seseli (Fam. Umbelliferae)" Ars Pharm., 31(34), 241-256 (1990).

13. Kumar, R., Gupta, B.D., Banerjee, S.K., Atal, C.K. "New Coumarins from Seseli sibiricum" Phytochemistry, 17, 2111-2114 (1978).

14. Banerjee, S.K., Gupta, B.D., Kumar, R., Atal, C.K. "New Coumarins from the Umbels of Seseli sibiricum" Phytochemistry, 19, 281-284 (1980).

15. Banerjee, S.K., Mukhopadhay, S., Gupta, B.D., Singh, K., Raj, S. "Sesebrinic Acid, A Cinnamic Acid Derivative from Seseli sibiricum" Phytochemistry, 26(6), 1817-1820 (1987).

16. Austin, P.W., Seshadri, T.R., Sood, M.S., Vishwapaul, M.S. "Components of Seseli sibiricum; Constitution and Synthesis of Sibiricin, a New Coumarin" Tetrahedron, 24, 3247-3253 (1968).

17. Kapoor, S.K., Sharma, Y.N., Kidwai, A.R. "Chemical Investigation of Seseli sibiricum" Phytochemistry, 7, 147-149 (1968). 
18. Bellino, A., Venturella, P., Marino, M.L., Servettaz, O., Venturella, G. "Coumarins from Seseli bocconi" Phytochemistry, 25(5), 1195-1199 (1986).

19. Ceccherelli, P., Curini, M., Marcotullio, M.C., Madruzza, G "Tortuoside, A New Natural Coumarin Glucoside from Seseli tortuosum" J. Nat. Prod., 53(2), 536-538 (1990).

20. Glowniak, K., DoraczyOska-Szopa, A., Erkelens, C, Van Der Sluis, W.G. "Isopeucenidin and Libanotin from Libanotis intermedia Roots: Isolation and NMRAnalysis" Planta Med., 57, Supplement Issue 2., A 52 (1991).

21. Coassini Lokar, L.R., Delben, S. "Photoactive Furocoumarins in Two Populations of Seseli elatum" Phytochemistry, 27(4), 1073-1077 (1988).

22. Lemmich, J., Shabana, M. "Coumarin Sulphates of Seseli libanotis" Phytochemistry, 23(4), 863-865 (1984).

23. Nielsen, B.E., Larsen, P.K., Lemmich, J. "Constituens of Umbelliferous Plants, XIII. Coumarins from Seseli gummiferum Pall. The Structure of Three New Coumarins" Acta Chem. Scand., 24(8), 2863-2867 (1970).

24. Murray, R.D.H., Mendez, J., Brown, S.A. The Natural Coumarins, Occurence, Chemistry and Biochemistry., John Wiley \& Sons Ltd., New York (1982).

25. Lemmich, J., Lemmich, E., Nielsen, B.E. "Constituens of Umbelliferous Plants, VIII. Coumarins from the Root of Seseli libanotis (L.) Koch. The Structure of Three New Coumarins" Açta Chem. Scand., 20(9), 2497-2507 (1966).

26. Gonzalez, A.G., Barroso, J.T., Lopez-Dorta, H., Luis, J.R., Rodriguez-Luis, F. "Pyranocoumarin Derivatives from Seseli tortuosum" Phytochemistry, 18, 1021-1023 (1979).

27. Nielsen, B.E., Larsen, P.K., Lemmich, J. "Constituents of Umbelliferous Plants, XVII. Coumarins from Seseli gummiferum Pall. The Structure of Two New Coumarins" Acta Chem. Scand., 25(2), 529-533 (1971).

28. Barrero, A.F., Herrador, M.M., Arteaga, P. "Sesquiterpenes and Phenylpropanoids from Seseli vayredanum" Phytochemistry, 31(1), 203-207 (1992).

29. Barrero, A.F., Herrador, M.M., Arteaga, P. "Sesquiterpene Lactones and Others Constituents of Seseli vayredanum" Phytochemistry, 37(5), 1351-1358 (1994).

30. Coassini Lokar, 1., Moneghini, M., Mellerio, G. "Taxonomical Studies on Seseli elatum L. and Allied Species. 2. Essential Oil Variation Among Three Populations" Webbia, 40(2), 279-288 (1986).

31. Başer, K.H.C., Özek, T., Nuriddinov, Kh.R., Nigmatullaev, A.M., Khadzimatov, K.Kh., Aripov, Kh.N. "Essential Oils of Mediasia macrophylla (Regel et Schmalh.) Pimen. and Foeniculum vulgare Mili. from Uzbekistan" J. Essent. Oil Res., 9, 249-250 (1997).

32. Başer, K.H.C., Özek, T., Kürkçüoğlu, M., Aytaç, Z. "Essential Oil of Seseli campestre Besser" /. Essent. Oil Res., 1, 105-107 (2000).

33. Kaya, A., Demirci B., Başer K.H.C., "The Essential Oil Composition of Seseli tortuosum L. Growing in Turkey" Flavour and Fragrance Journal, 18, 159-167 (2003). 
34. Muckensturm, B., Diyani, F., Reduron, J.-P., Hildenbrand, M. "7Demethylplastochrome nol-2 and 2-Demethylplastoquinone-3 from Seseli farreynii" Phytochemistry, 45(3), 549-550 (1997).

35. Chaturvedi, R.V., Tripathi, S.C. "Fungitoxic, Physico-Chemical and Phytotoxic Properties of Essential Oil of S. indicum W. \& A." /. Phytopathology, 124, 316-322 (1989).

36. Huang, L., Kashiwada, Y., Cosentino L. M., Fan, S., Chen, C.-FL, Mcphail, A. T., Fujioka, T., Mihashi, K., Lee, K.-H. "Anti-AIDS Agents. 15. Synthesis and Anti-HIV Activitiy of Dihydroseselins and Related Analogs" /. Med. Chem., 37, 3947-3955 (1994).

37. Wall, M.E., Wani, M.C., Manikumar, G., Hughes, T J., Taylor, H., Mc Givney, R., Warner, J. "Plant Antimutagenic Agents, 3. Coumarins" /. Nat. Prod., 51(6), 11481152(1988).

38. Magiatis, P., Melliou, E., Skaltsounis, A.-L., Mitaku, S., Leonce, S., Renard, P., Pierre, A., Atassi, G. "Synthesis and Cytotoxic Activity of Pyranocoumarins of the Seselin and Xanthyletin Series" /. Nat. Prod. 61, 982-986 (1998).

39. Şener, B., Mutlugil, A. "Doğal Kumarinler, Kimyasal Yapıları ve Biyolojik Aktiviteleri" FABAD J. Pharm. Sci., 12, 99-114 (1987).

40. Egan, D., O'Kennedy, R., Moran, E., Cox, D., Prosser, E., Thornes, R.D. "The Pharmacology, Metabolism, Analysis, and Applications of Coumarin and Coumarin Related Compounds" Drug Metab. Rev., 22(5), 503-529 (1990).

41. Hu, C.-Q., Chang, J.-J., Lee, K.-H. "Antitumor Agents, 115. Seselidiol, A New Cytotoxic Polyacetylene from Seseli mairei" J. Nat. Prod., 53(4), 932-935 (1990).

42. Tandan, S.K., Chandra, S., Tripathi, H. C, Lal, J. "Pharmacological Actions of Seselin, A Coumarin from Seseli indicum Seeds" Fitoterapia, LXI (4), 360-363 (1990)

43. Garcia-Argâez, A.N., Ramirez Apan, T.O., Delgado H.P., Velâzquez, G., Martınez-Vazquez, M. "Anti-Inflammatory Activity of Coumarins Decatropis bicolor on TPA Ear Mice Model" Planta Med., 66, 279-281 (2000).

44. Willette, R.E., Soine, T.O. "Isolation, Purification, and Structure Determination of Pteryxin and Suksdorfin" J. Pharm. Sci., 51(2), 149-156 (1962).

45. Kozawa, T., Sakai, K., Uchida, M., Okuyama, T., Shibata, S. "Calcium Antagonistic Action of a Coumarin Isolated from "Qian-Hu", A Chinese Traditional Medicine" J. Pharm. Pharmacol, 33, 317-320 (1980).

46. Ojala, T., Vuorela, P., Törnquist, K. "The Coumarin Osthol Attenuates the Binding of Thyrotropin-Releasing Hormone in Rat Pituitary GH4C1 Cells" Planta. Med., 67, 236239(2001).

47. Pathak, M.A., Joshi, P.C. "The Nature and Molecular Basis of Cutaneous Photosensitivity Reactions to Psoralens and Coal Tar" /. Invest. Derm., 80, 066s-074s (1983).

48. Berdahl, D.R., Wasserman, H.H "The Reaction of Psoralens and Related Benzofurans with Singlet Oxygen An Account of Current Research" Israel Journal of Chemistry, 23, 409-414(1983). 
49. Dubertret, L., Averbeck, D., Bisagni, E., Moron, J., Moustacchi, E., Billardon, C, Papadopoulo, D., Nocentini, S., Vigny, P., Blais, J., Bensasson, R.V., RonfardHaret, J.C., Land, E.J., Zajdela, F., Latarjet, R. "Photochemotherapy Using Pyridopsoralens" Biochimie, 67,417-422 (1985).

50. Rodighiero, G., Dall'acqua, F. "Present Aspects Concerning the Molecular Mechanisms of Photochemotherapy vvith Psoralens" Drug Exptl. Clin. Res., XII (6/7), 507-515(1986).

51. Şener, B., Mutlugil, A. "Doğal Furanokumarinler ve Fototoksik Aktivite" FABAD J. Pharm. Sci., 12, 243-249 (1987).

52. Dixit, V., Chaturvedi, R.V., Tripathi, S.C. "Evaluation of Some Essential Oils Against Pulse Bettle (Callosobrucus chinensis)" Nat. Acad. Sci. Letters, 15(8), 255-257 (1992).

53. Baytop, T., Türkiye'de Bitkiler ile Tedavi. İlaveli İkinci Baskı, Nobel Tıp Kitabevi, Ankara, s.375 (1999).

54. Bintsis, T., Litopoulou-Tzanetaki, E., Davies, R., Robinson, R.K. "The Antimicrobial Effects of Long-Wave Ultra-Violet Light and Furocoumarins on Some Micro-organisms that Occur in Cheese Brines" Food Microbiology, 17, 687-695 (2000).

55. Kawasaki C, Okuyama, T., Shibata, S., Iitaka, Y. "Studies on Coumarins of a Chinese Drug "Qian-Hu" ; VI. Coumarins of Angelica edulis" Planta Med., 50, 492-496 (1984).

56. Kawase, M., Varu, B., Shah, A., Motohashi, N., Tani, S.; Saito, S., Debnath, S., Mahapatra, S., Dastıdar, S.G., Chakrabarty, A. N. "Antimicrobial Activity of New Coumarin Derivatives" Arzneim.-ForschJDrug Res., 51(1), 67-71 (2001).

57. Mizuno, A., Takata, M., Okada, Y., Okuyama, T., Nishino, H., Takayasu, J., Ivrashima, A. "Structures of New Coumarins and Antitumour-Promoting Activity of Coumarins from Angelica edulis" Planta Med., 60, 333-336 (1994).

58. Thastrup, O., Fjalland, B., Lemmich, J. "Coronary Vasodilatory, Spasmolytic and cAMP-Phosphodiesterase Inhibitory Properties of Dihydropyranocoumarins and Dihydrofurano coumarins" Açta Pharmacol. et Toxicol., 52, 246-253 (1983).

59. Ojala, T., Remes, S., Haansuu, P., Vuorela, H., Hitunen, R., Haahtela, K., Vuorela, P. "Antimicrobial Activity of Some Coumarin Containing Herbal Plants Growing in Finland" /. Ethnopharmacology, 73,299-305 (2000).

60. Matsuda, H., Murakanıi, T., Nishida, N., Kageura, T., Yoshikawa, M. "Medicinal Foodstuffs. XX." Vasorelaxant Active Constituents from The Roots of Angelica furcijuga Kitagawa: Structures of Hyuganins A, B, C, and D" Chem. Pharm. Bull., 48(10), 1429-1435 (2000)

61. Baytop, T., Türkçe Bitki Adları Sözlüğ̈̈. Atatürk Kültür, Dil ve Tarih Yüksek Kurumu, TDKY 3578, Ankara, TTK Basımevi, s.169 (1994) 


\section{ANKARA ÜNIVERSITESİ ECZACILIK FAKÜLTESİ DERGİSİ 2003 DİZINI \\ JOURNAL OF FACULTY OF PHARMACY OF ANKARA UNIVERSITY \\ INDEX FOR 2003 \\ YAZAR DİZINİ \\ AUTHOR INDEX}

A

AKGÜN, DAR, Kadriye: 32(3); 143-150,2003

AKIN, Ahmet: 32(3); 151-157,2003

ALTANLAR, Nurten: 32(3); 151-157, 2003, 32(3); P 159-163,2003

ATASEVER, Belkis: 32(3); 143-150,2003

ATICI, Tahir: 32(1); 19-29,2003

\section{B}

BAHADIR, Özlem: 32(1); 55-92,2003

BAYKARA, Tamer: 32(2); 125-142,2003

BOZKIR, Asuman: 32(3); 175-193,2003

CALISKAN, Deniz: 32(3); 193-206, 2003

CITOGLU, SALTAN, Gülcin: 32(1); 31-35, 2003 , $32(2)$; 93-97,2003,32(3); 159-163,2003

DOĞRU, PEKİNER, Bilgehan: 32(4); 35-59,2003

\section{E}

EKE,CAN,Benay: 32(1); 1-18,2003

ERDEM, KURUCA, Serap: 32(3); 143-150,2003

ERDEN, ÇALIŞIR, Zeynep: 32(3); 193-206,2003

\section{G}

GÖNENC, Aymelek: 32(4); 13-22,2003

GÜRBÜŽ, AKARSU, Burçak: 32(4); 1-4,2003

GÜRCAN, Safa: $32(4) ; 13-22,2003$

GÜRKAN, Elçin: 32(4); 1-4,2003

G ÜVEN D I K, Gülin: 32(4); 5-11,2003

\section{K}

KARABAY, Ülkü: 32(2); 113-120,2003

KARAMENDERES, Canan: 32(2); 113-120,2003

KIZILAY, AKYÜZ,Çiğdem: 32(1); 31-35, 2003, 32(2) 121-124 2003

KONUKLUGiL, Belma: 32(1); 55-92,2003

$\mathrm{M}$

MERIÇLİ, Ali: 32(3); 143-150,2003

$\mathrm{N}$

NEBİOĞLU, Serpil: 32(3); 207-208,2003

O

ONAT, Ayhan: 32(4); 13-22,2003

ORHAN, İlkay: 32(1); 19-29,2003

OZANSOY, Gülgün: 32(4); 23-33,2003 o

ÖZKAL, Nazire: 32(4); 61-76,2003

$P$

PEKINNER,DOĞRU, Bilgehan: 32(2); 99-112,2003

$\mathrm{S}$

SAATCi,Feray: 32(3); 175-193,2003

SEYHANLI, Vildan: 32(3); 143-150,2003

SEZGIN, Zerrin: 32(2); 125-142,2003

SOYOĞUL, GÜRER, Umran: 32(4); 1-4,2003

ŞENER, Bilge: 32(1); 19-29,2003

$T$

TEKİNER,GÜLBAS,Betiil P.: 32(3); 165-173,2003

TORUN, Meral: 32(4); 13-22,2003

TOSUN, Alev: 32(4); 61-76,2003

TOSUN,Fatma: 32(1); 31-35, 2003, 32(2); 121-124, 2003

TURAN, Nevruz: $32(3) ; 143-150,2003$

TUZLACI, Ertan: 32(4); 1-4,2003

$\mathbf{U}$

ULUSOYLU, Melek: 32(4); 1-4,2003

$\mathbf{Y}$

YILDIZ,ÖREN,İlkay: 32(3); 165-173,2003

YILMAZ, Ayşegül: 32(4); 5-11,2003

YILMAZ, Betül: 32(1); 37-53,2003,32(2); 93-97, 2003

YÜCEL, Nihal: 32(3); 151-157,2003

YÜKSEL, Nilüfer: 32(2); 125-142,2003

WIESPONGPAND,Puntip: 32(1)；19-29,2003

$\mathrm{Z}$

ZEYBEK,Ulvi: 32(2); 113-120,2003 


\section{KONU DİZINI}

A

Achillea setacea: 32(2); 113-120,2003

AİDS: $32(1)$; 55-92,2003

Akciğer: $32(1) ; 1-18,2003$

Akut Lenfoblastik Lösemi: 32(3)；143-150, 2003

Alg: 32(1); 19-29,2003

Amfifilik ko- polimerler: 32(2); 125-142,2003

Antialgal aktivite: $32(1)$; $19-29,2003$,

Antibakteriyel aktivite: 32(2); 93-97, 2003, 32(3); 159-163, 2003, 32(4); 1-4, 2003

Antibiyotik duyarlılı̆̆ı: 32(3); 151-157,2003

Antifungal aktivite: 32(2); 93-97, 2003, 32(3);

2003

Antimikrobiyal aktivite: $32(2)$; 113-120,2003

Antimikrobiyal etki: $32(3)$; 165-173,2003

Anti-HIV aktivite: 32(1); 55-92, 2003

Antioksidan: 32(4); 35-59,2003

Antrakinonlar: $32(1) ; 31-35,2003$

Asteraceae: 32(2); 113-120,2003

B

Ballota: 32(1); 37-53,2003, 32(2); 93-97, 2003

Becke3LYP/6-31G(d,p): 32(3); 165-173,2003

Benzoksazinler: 32(3); 165-173,2003

Biyoadezif mikropartiküler sistem: 32(3); 175-193,2003

Biyolojik aktivite: 32(4); 61-76, 2003

Brine shrimp: 32(4); 1-4, 2003

Böbrek: 32(1); 1-18,2003

Bulunma sıklığı: 32(3); 151-157,2003

\section{C}

1,8 cineole: $32(2) ; 113-120,2003$

Constrast agent: $32(2) ; 125-142,2003$

D

DFT:32(3); 165-173,2003

DIMBOA: 32(3); 165-173,2003

Demir: 32(4); 13-22,2003

Diterpen: $32(1) ; 37-53,2003$

Diyabet: $32(4) ; 23-33,2003$

\section{E}

Etki: 32(3); 193-206,2003

\section{F}

Feniletanoit: $32(1) ; 37-53,2003$

Ferritin: 32(4); 13-22, 2003

Flavonoidler: $32(1) ; 31-35,2003,32(2)$

32(3); 143-150,2003

Flavonoit: $32(1) ; 37-53,2003$

Fosfolipid: 32(2); 99112, 2003

G

Gonocytisus ptrocladus: $32(2) ; 121-124,2003$

$\mathrm{H}$

Hareketli aeromonas türleri: 32(3); 151-157,2003

Hava kirliliği: $32(4) ; 5-11,2003$

Hipertiroidizm: 32(4); 13-22,2003

Hipotiroidizm: 32(4); 13-22,2003

I

İlaç hedefleme: 32(2); 125-142,2003

İlaç taşıyıcı sistemleri: $32(2) ; 125-142,2003$

İn vitro sitotoksisite: $32(3)$; 143-150, 2003
Juglans regia: 32(3); 159-163,2003

K

Kamazulen: 32(2); 113-120,2003

Karboksihemoglobin: 32(4); 5-11,2003

Karbonmonoksit: 32(4); 5-11,2003

Katk1 maddesi: 32(3); 193-206,2003

Kimyasal bileşim: 32(4); 61-76, 2003

Kontrast ajan: 32(2); 125-142,2003

Kritik misel konsantrasyonu: 32(2); 125-142 159-163, 2003

Kuyu suları: 32(3); 151-157,2003

L

Lamiaceae: 32(1); 37-53, 2003, 32(2); 93-97

2003

Lythrum salicaria: 32(3); 159-163,2003

M

Mikrozom: 32(1); 1-18,2003

Monooksijenaz: 32(1); 1-18,2003

Mukozal adjuvan: 32(3); 175-193,2003

$\mathrm{N}$

NALT: $32(3) ; 175-193,2003$

Nazal aşı: 32(3); 175-193,2003

$\mathrm{O}$

Ononis spinosa: 32(3); 159-163,2003

P

Plantago major: 32(3); 159-163,2003

Plazma: 32(2); 99112,2003

Pisisidal aktivite: $32(1) ; 19-29,2003$

Polimerik miseller: 32(2); 125-142,2003

$\mathrm{R}$

Rheum ribes: $32(1) ; 31-35,2003$

Rubus sanctus: 32(4); 1-4,2003

$\mathrm{S}$

Saponozit: 32(1); 37-53, 2003

Seseli L.: 32(4); 61-76,2003

Siçan: 32(1); 1-18,2003,32(4); 23-33, 2003

Sistem: 32(3); 193-206, 2003

Spektrofotometri: 32(4); 5-11,2003

121-124, 2003, Su bitkisi: 32(1); 19-29,2003

$\mathrm{T}$

Tanen: 32(1); 37-53, 2003

o-Tokoferol: 32(4); 35-59, 2003

Trakea: $32(4) ; 23-33,2003$

Triterpen: $32(4) ; 1-4,2003$

Tuzlu su karidesi toksisitesi: 32(1); 19-29 2003

Türler: 32(2); 99112,2003

$\mathrm{U}$

Uçucu yağ: 32(1); 37-53, 2003, 32(2); 113-120 2003

Umbelliferae: 32(4); 61-76,2003

V

Vitamin E: 32(4); 23-33, 2003, 32(4); 35-59 2003

Y

Yağ asitleri: 32(2); 99112, 2003

Yiyecek: 32(3); 193-206, 2003 


\section{SUBJECT INDEX}

A

Achillea setacea: 32(2); 113-120,2003

Acute lymphoblastic leukemia: 32(3); 143-150, 2003

Additive: 32(3); 193-206,2003

AIDS: $32(1) ; 55-92,2003$

Air pollution: $32(4) ; 5-11,2003$

Algae:32(1); 19-29,2003

Amphiphilic co- polymers: $32(2) ; 125-142,2003$

Anthraquinones: $32(1) ; 31-35,2003$

Antialgal activity: $32(1) ; 19-29,2003$

Antibacterial activity: 32(2); 93-97, 2003, 32(3); 159-163, 2003,32(4); 1-4,2003

Antifungal activity: 32(2); 93-97, 2003, 32(3); 159-163, 2003

Anti-HIV activity: 32(1); 55-92,2003

Antimicrobial activity: 32(2); 113-120, 2003, 32(3); 165173,2003

Antibiotic susceptibiiity: 32(3); 151-157, 2003

Antioxidant: $32(4) ; 35-59,2003$

Aquatic plant: 32(1); 19-29, 2003

Asteraceae: 32(2); 113-120, 2003

B

Ballota: 32(1); 37-53,2003,32(2); 93-97,2003

Becke3LYP/6-31G(d,p): 32(3); 165-173,2003

Benzoxazines: 32(3); 165-173, 2003

Bioadhesive microparticulate system: $32(3) ; 175-193$ 2003

Biological activity: 32(4); 61-76, 2003

Brine shrimp: 32(4); 1-4, 2003

Brine shrimp toxicity: 32(1); 19-29,2003

C

1,8 cineole: $32(2) ; 113-120,2003$

Carbonmomoxide: 32(4); 5-11,2003

Carboxyhemoglobin: 32(4); 5-11,2003

Chamazulene: 32(2); 113-120,2003

Chemical constituent: 32(4); 61-76, 2003

Contrast agent: $32(2) ; 125-142,2003$

Critical micelle concentration: $32(2) ; 125-142,2003$

D

DFT:32(3); 165-173,2003

DIMBOA: $32(3) ; 165-173,2003$

Diabetes: 32(4); 23-33, 2003

Diterpene: 32(1); 37-53,2003

Drug carrier system: 32(2); 125-142,2003

Drug targeting: $32(2) ; 125-142,2003$

E

Effect: $32(3) ; 193-206,2003$

Essential oil: $32(2) ; 113-120,2003$

F

Fatty acids: $32(2) ; 99112,2003$

Ferritin: 32(4); 13-22,2003

Flavonoid: $32(1) ; 37-53,2003$

Flavonoids: $32(1) ; 31-35,2003,32(2) ; 121-124,2003$, 32(3); 143-150,2003

Food: 32(3); 193-206,2003

G

Gonocytisus ptrocladus: 32(2); 121-124,2003

H

Hyperthyroidism: 32(4); 13-22,2003

Hypothyroidism: 32(4); 13-22, 2003
Incidence: 32(3); 151-157,2003

in vitro cytotoxicity: 32(3); 143-150, 2003

Iron:32(4); 13-22,2003

$\mathbf{J}$

Juglans regia: 32(3); 159-163,2003

$\mathrm{K}$

Kidney: 32(1); 1-18,2003

$\mathrm{L}$

Lamiaceae: 32(1); 37-53,2003

Lung:32(1); 1-18,2003

Lythrum salicaria: 32(3); 159-163,2003

$\mathrm{M}$

Microsomes: 32(1); 1-18,2003

Monooxygenases: 32(1); 1-18,2003

Motile aeromonas spp.: 32(3); 151-157, 2003

Mucosal adjuvants: 32(3); 175-193,2003

$\mathrm{N}$

Nasal vaccine: $32(3) ; 175-193,2003$

$\mathrm{O}$

Ononis spinosa: 32(3); 159-163,2003

$\mathrm{P}$

Phenylethonoid: 32(1); 37-53,2003

Phospholipid: 32(2); 99112,2003

Plantago major: 32(3); 159-163,2003

Plasma: 32(2); 99112,2003

Polymeric micelles: 32(2); 125-142,2003

Psicicidal activity: 32(1); 19-29,2003

$\mathrm{R}$

Rat: 32(1); 1-18,2003,32(4); 23-33,2003

Rheum: 32(1); 31-35,2003

Rubus sanctus schreber: 32(4); 1-4,2003

$\mathrm{S}$

Saponin: 32(1); 37-53,2003

Seseli L.: 32(4); 61-76, 2003

Species: 32(2); 99112,2003

Spectrophotometry: 32(4); 5-11,2003

System: 32(3); 193-206,2003

$\mathrm{T}$

Tannin: 32(1); 37-53, 2003

'=-Tocopherol: 32(4); 35-59, 2003

Trachea: 32(4); 23-33, 2003

Triterpenoid: $32(4) ; 1-4,2003$

$\mathrm{U}$

Umbelliferae: 32(4); 61-76, 2003

Untreated well water: 32(3); 151-157,2003

V Vitamin E: 32(4); 23-33, 2003, 32(4); 35-59, 2003

Volatile oils: 32(1); 37-53, 2003 